\section{Über die Ursache der Supraleitung *}

\section{H. KREBS}

Laboratorium für Anorganische Chemie der Universität Stuttgart (TH)

(Z. Naturforsch. 23 a, 332-333 [1968] ; eingegangen am 9. Dezember 1967)

The occurance of superconductivity appears to be connected with the simple requirement that the electrons of a band have the possibility of moving in at least one direction without crossing plane or conical atomic nodal surfaces. This hypothesis is arrived at from a consideration of the bonding relationships in superconducting compounds.

Die elektrischen Eigenschaften und die Struktur kristalliner, glasiger und flüssiger Phasen sind weitgehend bestimmt durch die Ausdehnung der Orbitale der Valenzelektronen, ihre Geometrie und ihre Besetzung mit Valenzelektronen ${ }^{1}$.

Darüber hinaus scheint auch das Auftreten der Supraleitung mit der Tatsache verknüpft zu sein, daß Orbitale eines Bandes so überlappen, daß die Elektronen die Möglichkeit haben, sich wenigstens in einer Raumrichtung so zu bewegen, daß sie keine ebene oder kegelförmige atomare Knotenfläche zu durchsetzen brauchen. Der einfachste Fall liegt dann vor, wenn s-Orbitale in einem Gitter ein eigenes und von anderen energetisch getrenntes Band bilden.

\begin{tabular}{|c|c|c|c|c|c|}
\hline \multicolumn{3}{|c|}{ NaCl-Struktur } & \multicolumn{3}{|c|}{$\mathrm{CaF}_{2}-$ Struktur } \\
\hline \multirow{6}{*}{$\begin{array}{l}\mathrm{ScN} \\
\mathrm{TiC} \\
\mathrm{ZrC} \\
\mathrm{Zr}_{0,74} \mathrm{Nb}_{0,26} \mathrm{C} \\
\mathrm{Zr}_{0,5} \mathrm{Nb}_{0,5} \mathrm{C} \\
\mathrm{Zr}_{0,23} \mathrm{Nb}_{0,77} \mathrm{C} \\
\mathrm{NbC}\end{array}$} & 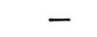 & \multirow{6}{*}{$\begin{array}{l}0 \\
0 \\
0 \\
+0,26 \\
+0,5 \\
+0,77 \\
+1\end{array}$} & $\mathrm{CoSi}_{2}$ & 1,22 & -1 \\
\hline & - & & PtGa2,33 & 2,9 & -1 \\
\hline & - & & $\mathrm{NiSi}_{2}$ & - & \\
\hline & 3,6 & & $\mathrm{PtAl}_{2}$ & - & -2 \\
\hline & 5,9 & & $\mathrm{AuAl}_{2}$ & - & -1 \\
\hline & $\begin{array}{r}9,3 \\
12,0\end{array}$ & & \multicolumn{3}{|c|}{ Pyrit-Struktur } \\
\hline \multirow{12}{*}{$\begin{array}{l}\text { TiN } \\
\text { ZrN } \\
\text { HfN } \\
\mathrm{NbC}_{0} \\
\text { TiO } \\
\text { VN } \\
\mathrm{NbN}_{0} \\
\mathrm{NbN} \\
\mathrm{VO} \\
\mathrm{NbO} \\
\mathrm{UC} \\
\mathrm{UN}\end{array}$} & \multirow{2}{*}{$\begin{array}{l}9-5,6 \\
9-9,1\end{array}$} & \multirow{4}{*}{$\begin{array}{l}+1 \\
+1 \\
+1 \\
+1,7\end{array}$} & \multirow{2}{*}{$\mathrm{RhTe}_{2}$} & \multirow{2}{*}{1,51} & \multirow{2}{*}{+1} \\
\hline & & & & & \\
\hline & \multirow{2}{*}{$\begin{array}{r}6,2 \\
178\end{array}$} & & PdSbSe & 1,0 & \multirow{2}{*}{$\begin{array}{l}+1 \\
+1\end{array}$} \\
\hline & & & PdSbTe & 1,2 & \\
\hline & - & \multirow{2}{*}{$\begin{array}{l}+2 \\
+2\end{array}$} & PdBiSe & 1,0 & $\begin{array}{l}+1 \\
+1\end{array}$ \\
\hline & $\begin{array}{r}-8,2 \\
15,6\end{array}$ & & $\begin{array}{l}\text { PdBiTe } \\
\text { PtBiSe }\end{array}$ & 1,2 & $\begin{array}{l}+1 \\
+1\end{array}$ \\
\hline & 15,6 & $\begin{array}{r}\approx+2 \\
+2\end{array}$ & PtBiTe & $\begin{array}{l}1,45 \\
1,15\end{array}$ & $\begin{array}{l}+1 \\
+1\end{array}$ \\
\hline & $-10,0$ & \multirow{2}{*}{$\begin{array}{l}+3 \\
+3\end{array}$} & $\mathrm{AuSb}_{2}$ & \multirow{2}{*}{$\begin{array}{l}0,59 \\
1,25\end{array}$} & +1 \\
\hline & - & & $\mathrm{PdSb}_{2}$ & & \\
\hline & \multicolumn{2}{|l|}{-} & \multicolumn{2}{|c|}{$\mathrm{CdJ}_{2}$-Struktur } & \\
\hline & & & $\mathrm{PdTe}_{2}$ & 1,53 & \\
\hline & & & & & \\
\hline
\end{tabular}

Tab. 1. Sprungtemperatur $\left({ }^{\circ} \mathrm{K}\right)^{2-5}$ und Zahl der formal überzähligen bzw. fehlenden Elektronen. - Supraleitung bisher nicht festgestellt.

* Erstmals in Teilen vorgetragen anläßlich der Hauptversammlung der Gesellschaft Deutscher Chemiker, Berlin, 18. - 23. 9. 1967.

1 Vgl. H. Krebs, Grundzüge der anorganischen Kristallchemie, F. Enke Verlag, Stuttgart 1968.

2 B. T. Matthias, T. H. Geballe u. V. B. Compton, Rev. Mod. Phys. 35, 1 [1963].

3 B. W. Roberts, in Intermetallic Compounds, hrsg. von J. H. Westвrook, John Wiley, New York 1967, S. 581 ff.
Die meisten Supraleiter sind Metalle oder Legierungen, über deren Bandstruktur man wenig weiß. Um diese Vermutung über die Ursache der Supraleitung zu stützen, greift man daher am besten auf solche metallisch leitende Verbindungen zurück, über deren Bindungsverhältnisse von den Erfahrungen der anorganischen Chemie her Aussagen möglich sind. Tab. 1 enthält eine nach Kristallstrukturen geordnete Zusammenstellung von Verbindungsgruppen, in denen Supraleitung beobachtet wird.

Bei den sogenannten Hartstoffen mit NaCl-Struktur tritt Supraleitung nur auf, wenn die Wertigkeit des Metallatoms um 1 bis 2 geringer ist, als der Gruppenwertigkeit entspricht. Greift man als Beispiel das TiN heraus, so kann man ein $\sigma$-Bindungssystem in Richtung der Würfelkanten der Elementarzelle konstruieren mit den Elektronenkonfigurationen $\mathrm{Ti}^{+}\left(\mathrm{d}_{\gamma}{ }^{2} \mathrm{~s}\right)^{3}$-Hybrid und $\mathrm{N} \mathrm{s}^{2} \mathrm{p}^{3}$. Die Aufladung des Ti-Atoms kann teilweise auf das N-Atom übertragen werden durch dative $\pi$-Bindungen zwischen den p-Elektronen am $\mathrm{N}$-Atom und leeren $\mathrm{d}_{\varepsilon}$-Zuständen am Ti. Für das letzte Valenzelektron steht dann ein bindender Zustand, gebildet aus den weit ausgedehnten Orbitalen Ti 4s und N 3s zur Verfügung, wodurch die obengenannte Bedingung erfüllt wird. Ein antibindender Zustand, gebildet aus Ti 4s und Orbitalen der Hauptquantenzahl 2 am N-Atom dürfte energetisch ungünstiger sein. In der Reihe ${ }^{5} \mathrm{Zr}_{1-x} \mathrm{Nb}_{\mathrm{x}} \mathrm{C}$ ist die Höhe der Sprungtemperatur ungefähr linear abhängig von der Zahl der Valenzelektronen pro Atom, d. h. von der Besetzung des s-Bandes.

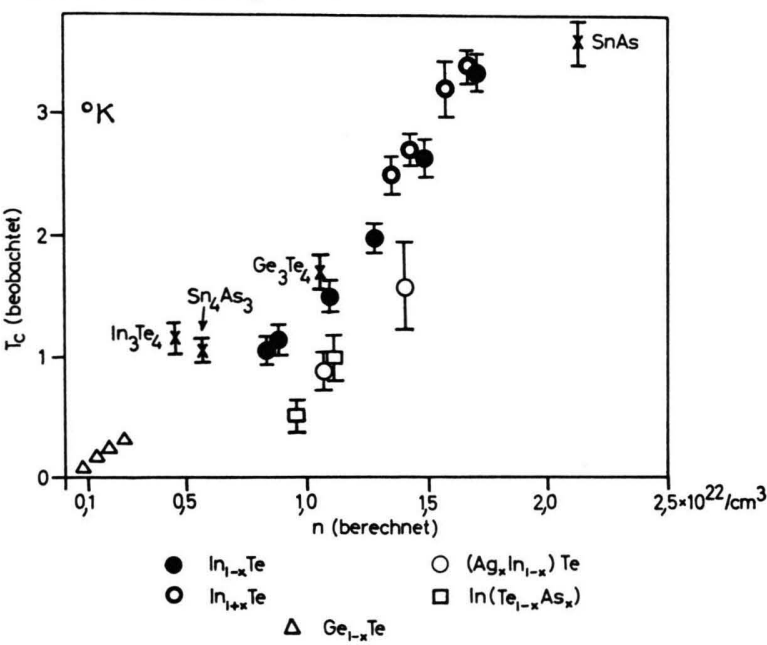

Abb. 1. Abhängigkeit der Sprungtemperatur von der Ladungsträgerkonzentration $n$ nach GELLER ${ }^{6}$, die der Zahl der einfach besetzten s-Zustände entspricht.

4 B. W. Roberts, in Progress in Cryogenics, Bd. IV, Heywood Book, London 1964, S. 159 ff.

5 John PIPer, Int. Symp. on Compounds of Interest in Nuclear Reactor Technology, 3-5, VIII, 1964, Boulder Cal., Hrsg. J. T. W Report No. 13, Edward Brothers Inc., Ann Arbor, Mich. 1964, S. $29 \mathrm{ff}$. 
In Abb. 1 ist die Sprungtemperatur von Halbmetallverbindungen mit Kochsalzstruktur in Abhängigkeit von der Konzentration von formal zweiwertigem In und von formal dreiwertigen Ge- oder Sn-Atomen wiedergegeben ${ }^{6}$. Diese experimentellen Ergebnisse von GeLLER, die der Ausgangspunkt unserer eigenen Utberlegungen waren, sind eine starke Stütze für die obige Annahme eines s-Bandes, da erstens kein Zweifel an der mehr oder weniger starken Besetzung von s-Zuständen der Metallatome In, Ge oder Sn besteht, und zweitens die Zahl der einfach besetzten s-Zustände die Höhe der Sprungtemperatur weitgehend bestimmt. Die Geometrie des Gitters ist bedingt durch die Symmetrie der p-Orbitale des Metall- wie des Nichtmetallatoms, die mesomere $\sigma$-Bindungen entlang den Kanten der Elementarzelle bilden ${ }^{1}$.

Die beiden einzigen supraleitenden Verbindungen mit Fluorit-Struktur sind das $\mathrm{CoSi}_{2}$ und das $\mathrm{PtGa}_{2,33} \mathrm{mit}$ jeweils 17 Valenzelektronen. Bei beiden Verbindungen fehlt an der Edelgasschale des Metallatoms ein Elektron. Dies kann ein s-Elektron sein.

Verschiedene supraleitende Verbindungen mit PyritStruktur sind bekannt ${ }^{7}$. Abgesehen vom $\mathrm{PdSb}_{2}$ besitzen alle ein Elektron mehr, als zur Auffüllung der Edelgasschalen benötigt wird. Die Entstehung dieser Verbindungen ist wohl auf die außerordentlich starke Tendenz der Metallatome zurückzuführen, stabile low spin-Komplexe zu bilden. Das überzählige Elektron wird in Kauf genommen und dürfte s-Zustände über der jeweiligen Edelgasschale der Atome besetzen.

Die Annahme der oben geschilderten Bedeutung eines s-Bandes für die Entstehung der Supraleitung wird auch noch durch folgende experimentelle Beobachtungen gestützt.

Supraleitung findet man häufiger bei Verbindungen, die schwere Elemente enthalten wie zum Beispiel Nb, Mo und W. Die energetische Konkurrenz zwischen den s- und d-Zuständen ist bei diesen ausgeprägter als bei den leichten Elementen $\mathrm{Ti}, \mathrm{V}$ und $\mathrm{Cr}$, bei denen der s-Zustand energetisch bevorzugt ist und daher für die Bindung voll beansprucht wird.

Im Pd-Atom ist die energetische Bevorzugung der dOrbitale gegenüber dem s-Orbital besonders ausgeprägt, denn das freie Atom besitzt die Elektronenkonfiguration $4 \mathrm{~d}^{10} 5 \mathrm{~s}^{0}$ an Stelle von $3 \mathrm{~d}^{8} 4 \mathrm{~s}^{2}$ bei $\mathrm{Ni}$ und

6 S. Geller, Vorbericht zum Symposium on the Chemical Bond in Semiconductors, Minsk 28. 5. -3. 6. 1967, S. 71.

7 F. Hulliger u. J. Müller, Phys. Letters 5, 226 [1963].

8 J. Goubeau, persönliche Mitteilung.
$5 d^{9} 6 s^{1}$ bei Pt. Das Pd kann deshalb auch supraleitende Verbindungen wie $\mathrm{PdSb}_{2}$ und $\mathrm{PdTe}_{2}$ bilden ${ }^{8}$, obwohl die Zahl der Elektronen gerade passend ist, um die Edelgasschalen der am Aufbau der Verbindungen beteiligten Atome zu erreichen. Eine tiefere Begründung dürfte darauf hinauslaufen, daß die energetische $\mathrm{Be}$ nachteiligung des $5 \mathrm{~s}$-Zustandes eine weite Ausdehnung des 5 s-Orbitals zur Folge hat. Das Orbital muß daher im Gitter komprimiert werden. Dies wird dadurch erleichtert, daß ein Teil der 5s-Elektronen in das angeregte s-Band gebracht wird, das wir weiter oben diskutiert haben.

Hissch und Buckel ${ }^{9}$ haben vor Jahren gefunden, da $\beta$ amorphe Metallfilme supraleitend sein können, wenn sie durch Kondensation von Dämpfen bei Temperaturen des flüssigen Heliums erhalten werden. Der zuerst entdeckte Fall war der des $\mathrm{Bi}$, dann kamen hinzu Be, Ga und Filme aus Sn mit Zusatz von $10 \% \mathrm{Cu}$. Die s-Funktionen sind kugelsymmetrisch. Zur Entstehung eines sBandes ist eine Ordnung der Atome nicht erforderlich.

Die Regeln von MatThias ${ }^{10,3}$ über das Auftreten der Supraleitung besonders bei $\sigma$ - und Laves-Phasen und solchen vom $\beta \mathrm{W}$ - und $a \mathrm{Mn}$-Typ beruhen auf der Angabe günstiger Elektronenkonzentrationen pro Atom. Dies mag mit der Besetzung von s-Zuständen zusammenhängen und entspricht den Beobachtungen von GEL${ }_{\text {LER }}{ }^{6}$ und PIPER ${ }^{5}$. Die Laves-Phasen $\mathrm{KBi}_{2}, \mathrm{RbBi}_{2}$ und $\mathrm{CsBi}_{2}$ zeigen abweichend von anderen supraleitenden Laves-Phasen ein Verhältnis zwischen der Zahl der Elektronen und der Zahl der Atome von 3,67 bzw. von 2,33 , wenn man die beiden 6s-Elektronen des $\mathrm{Bi}$ nicht mitzählt, da sie sich an der Bindung nicht beteiligen.

Das Gerüst der Bi-Atome dürfte zusammengehalten werden durch „bananen“-förmig verbogene $\sigma$-Bindungen von p-Elektronen, die jeweils in ähnlicher Weise nach zwei Seiten binden wie in den unter hohem Druck im primitiv kubischen Gitter kristallisierenden Elementen $\mathrm{P}$ und $\mathrm{Sb}^{1}$. Die Alkali-Atome besetzen Hohlräume des Gitters und werden so in eine diamantartige Anordnung gezwungen. Die Alkalimetalle selbst sind nicht supraleitend, da ihre kubisch innenzentrierte Struktur nahelegt anzunehmen, daß die Bindung durch p-Elektronen zustande kommt. In einer diamantartigen Anordnung der Atome entfällt aber der Grund für die Bevorzugung der p-Orbitale.

9 R. Hilsch, Proc. Int. Conf. on Low Temp. Physics, Hrsg. R. Bowers, Oxford 1951, S. 119. - W. Buckel u. R. Hilsch, Z. Phys. 138, 109 [1954].

10 B. T. Matthias, Progr. in Low Temp. Physics, Bd. II, S. 138, Interscience, New York 1955; Phys. Rev. 97, 74 [1955]. 